\title{
Cloning, sequencing, and expression of the P-protein gene (phe A) of Pseudomonas stutzeri in Escherichia coli: implications for evolutionary relationships in phenylalanine biosynthesis
}

\author{
RANDy S. Fischer, ${ }^{*}$ Genshi ZhaO and Roy A. Jensen \\ Department of Microbiology and Cell Science, University of Florida, Gainesville, FL 32611, USA
}

(Received 1 October 1990; revised 11 February 1991; accepted 27 February 1991)

\begin{abstract}
The phe $A$ gene encoding the bifunctional P-protein (chorismate mutase: prephenate dehydratase) was cloned from Pseudomonas stutzeri and sequenced. This is the first gene of phenylalanine biosynthesis to be cloned and sequenced from Pseudomonas. The phe A gene was expressed in Escherichia coli, allowing complementation of an $E$. coli phe $A$ auxotroph. The enzymic and physical properties of the P-protein from a recombinant $E$. coli auxotroph expressing the phe $A$ gene were identical to those of the native enzyme from $P$. stutzeri. The nucleotide sequence of the $P$. stutzeri phe $A$ gene was 1095 base pairs in length, predicting a 365-residue protein product with an $M_{\mathrm{r}}$ of 40844. Codon usage in the $P$. stutzeri phe $A$ gene was similar to that of Pseudomonas aeruginosa but unusual in that cytosine and guanine were used at nearly equal frequencies in the third codon position. The deduced P-protein product showed sequence homology with peptide sequences of the $E$. coli $\mathrm{P}$-protein, the $\mathrm{N}$-terminal portion of the $E$. coli $\mathrm{T}$-protein (chorismate mutase: prephenate dehydrogenase), and the monofunctional prephenate dehydratases of Bacillus subtilis and Corynebacterium glutamicum. A narrow range of values (26-35\%) for amino acid matches revealed by pairwise alignments of monofunctional and bifunctional proteins possessing activity for prephenate dehydratase suggests that extensive divergence has occurred between even the nearest phylogenetic lineages.
\end{abstract}

\section{Introduction}

Micro-organisms are diverse in the arrangement and variety of enzymes comprising the terminal pathways of L-phenylalanine biosynthesis (Fig. 1). Bacillus subtilis, Corynebacterium glutamicum, and other Gram-positive bacteria synthesize phenylalanine via phenylpyruvate. The latter is produced from chorismate in consecutive steps catalysed by the enzymes chorismate mutase and PDT (Berry et al., 1987; Fazel \& Jensen, 1980; Pierson \& Jensen, 1974). In $B$. subtilis, the chorismate mutase reaction may be catalysed by a low-activity species carried on a bifunctional protein (also containing the catalytic site for DAHP synthase, the initial enzymic

Abbreviations: ADT, arogenate dehydratase; CDT, cyclohexadienyl dehydratase: CM-F, monofunctional chorismate mutase: DAHP, 7 phospho-2-dehydro-3-deoxy-D-arabino-heptonate: PDT, prephenate dehydratase.

The nucleotide sequence data reported in this paper have been submitted to GenBank and have been assigned the accession number M35545. step of the pathway which carries out the condensation of phosphoenolpyruvate and erythrose 4-phosphate to form DAHP) or by a monofunctional, high-activity species (CM-F). The relationship of these enzymes has been reviewed by Byng \& Jensen (1983). The coexistence of chorismate mutase and DAHP synthase on a bifunctional protein has also been reported for Brevibacterium flavum (Shiio \& Sugimota, 1979).

Escherichia coli, Pseudomonas stutzeri, and many other Gram-negative bacteria synthesize phenylalanine employing the foregoing enzymic steps, but utilize a bifunctional protein (P-protein) to catalyse the overall conversion of chorismate to phenylpyruvate (Ahmad \& Jensen, 1986). Other micro-organisms such as Pseudomonas diminuta use an entirely different route, the arogenate pathway (Zamir et al., 1985). In this case three monofunctional enzymes, $\mathrm{CM}-\mathrm{F}$, prephenate aminotransferase and ADT, catalyse steps mediating the chorismate-to-phenylalanine conversion. Pseudomonas aeruginosa exemplifies those organisms having a pathway arrangement whereby dual pathways to phenylalanine coexist. In addition to the P-protein, $P$. aeruginosa 


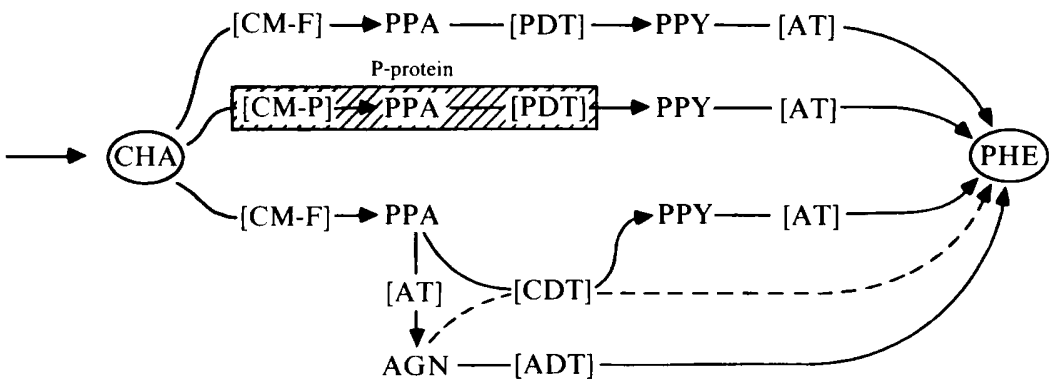

Fig. 1. Diversity of enzymic routes for the conversion of chorismate to L-phenylalanine. Abbreviations: CHA, chorismate; PPA, prephenate; PPY, phenylpyruvate; PHE, L-phenylalanine; AGN, L-arogenate; CM-F, 'free' chorismate mutase which is not a component of a bifunctional protein; CM-P, chorismate mutase component of the P-protein; PDT, prephenate dehydratase; CDT, cyclohexadienyl dehydratase which can utilize both PPA and AGN as substrate; ADT, arogenate dehydratase; AT denotes an unknown number of aminotransferases having overlapping substrate specificities and able to transaminate PPA or PPY in vitro. The CM-P and PDT activities are fused to form the $P$-protein, represented by the shaded rectangle. The route to PHE via AGN that is catalysed by the substrate-ambiguous CDT is depicted by the dashed line. has a broad-specificity CDT that can utilize either prephenate or L-arogenate as substrate (Patel et al., 1977). Another type of dual-pathway arrangement is found (unpublished data) in Zymomonas mobilis, which expresses two monofunctional substrate-specific dehydratases (ADT and PDT).

In organisms where the dehydratase activity is catalysed by a monofunctional PDT, ADT or CDT, a monofunctional CM-F is inevitably present (Ahmad \& Jensen, 1988a). We designate monofunctional chorismate mutase as $\mathrm{CM}-\mathrm{F}$ to distinguish it from the chorismate mutase components of the bifunctional proteins.

The complexity of phenylalanine biosynthesis in Pseudomonas is particularly intriguing since most of the pathway alternatives illustrated in Fig. 1 can be demonstrated in different pseudomonad species. The relative simplicity of phenylalanine biosynthesis in $P$. stutzeri, a close relative of $P$. aeruginosa in rRNA homology group I, has led to its choice for initial investigations aimed at delineation of the evolutionary relationships of genes encoding the monofunctional and bifunctional phenylalanine-pathway enzymes of parallel catalytic function in these Gram-negative organisms.

\section{Methods}

Bacterial strains, media and growth. The bacterial strains and plasmids utilized or constructed in this investigation are shown in Table 1. P. stutzeri was cultured as described by Carlson et al. (1983). E. coli strains were grown in M9 (Miller, 1972) minimal medium or LB (Davis et al., 1980a) medium at $37^{\circ} \mathrm{C}$. Amino acids $\left(50 \mu \mathrm{g} \mathrm{ml}^{-1}\right)$ and thiamin hydrochloride $\left(17 \mu \mathrm{g} \mathrm{m}^{-1}\right)$ were added as growth supplements when required. Antibiotics, when appropriate to provide selective pressure for plasmids, were added to the medium at standard concentrations (Maniatis et al., 1982). X-Gal (5-bromo-4-chloro-3indoxyl $\beta$-D-galactopyranoside) and IPTG (isopropyl $\beta$-D-thiogalactopyranoside) were included at concentrations recommended by the supplier (Promega) when recombinants were screened in pGEM-4Z or pGEM-5Z plasmids transformed into JM109 cells. Growth rates were determined by measuring turbidity of exponentially growing cultures at $600 \mathrm{~nm}$ in a Perkin-Elmer model 35 spectrophotometer.

DNA manipulation and genetic procedures. Standard molecular biology procedures were performed as described by Maniatis et al. (1982) unless otherwise indicated. Bacterial chromosomal DNA was purified by the method of Yuan \& Lin (1982). DNA for large-scale plasmid preparations was isolated by a Triton X-100 lysis procedure (Clewell \& Helinski, 1969; Kupersztoch \& Helinski, 1973) and purified by $\mathrm{CsCl} /$ ethidium bromide buoyant density gradient centrifugation (Humphreys et al., 1975). Small-scale plasmid isolation was performed according to the protocol of Davis et al. $(1980 \mathrm{~b})$. E. coli strains were transformed by use of a $\mathrm{CaCl}_{2}$ method (Dagert \& Ehrlich, 1979). Natural transformation of $P$. stutzeri JM706 was carried out as described by Carlson et al. (1983).

Cloning vectors, reagents, and enzymes for molecular biology. Restriction enzymes and T4 DNA ligase were obtained from New England BioLabs or Bethesda Research Laboratories. Calf intestinal alkaline phosphatase, pHC79, and the $\lambda$ DNA packaging kit were obtained from Boehringer Mannheim Biochemicals. X-Gal, IPTG, and the plasmids pGEM-4Z and pGEM-5Zf $(+)$ were purchased from Promega. Each product was used as recommended by the manufacturer.

Preparation and screening of the P. stutzeri gene bank. A genomic, randomly generated $P_{S t} \mathrm{I}$ gene bank was constructed in the cosmid vector pHC79 by partial methylation of $P$. stutzeri JM300 DNA followed by limit digestion. $P$. stutzeri was chosen for the cloning of the phe A gene because: (i) wild-type strain JM300 possesses only the bifunctional P-protein route to phenylalanine; (ii) strain JM706, a Phe ${ }^{-}$ derivative of JM300, demonstrates an absolute growth requirement for phenylalanine, has a low reversion frequency, and is deficient in prephenate dehydratase activity; and (iii) strain JM706 can be transformed to prototrophy with high frequency by natural transformation with homologous chromosomal DNA (Carlson et al., 1983, 1984), regardless of flanking sequences.

The gene library of $P$. stutzeri was prepared as described below to 
Table 1. Bacterial strains and plasmids

\begin{tabular}{|c|c|c|}
\hline $\begin{array}{c}\text { Strain } \\
\text { or plasmid }\end{array}$ & Genotype or description & Source or reference \\
\hline \multicolumn{3}{|l|}{ P. stutzeri } \\
\hline JM300 & Prototroph & Carlson et al. (1983). \\
\hline JM706 & $\begin{array}{l}\text { phe A mutant defective in prephenate dehydratase activity } \\
\text { (Byng et al., 1983) isolated from NTG mutagenesis of } \\
\text { JM300 }\end{array}$ & $\begin{array}{l}\text { J. L. Ingraham, Dept. of } \\
\text { Microbiology, Univ. of } \\
\text { California, Davis }\end{array}$ \\
\hline \multicolumn{3}{|l|}{ E. coli } \\
\hline $\mathrm{DHl}$ & gyrA96 recAl relAl endAl thi-1 hsdRI7 supE44 & CGSC* 6040 \\
\hline JP2255 & aroF363 phe $A 361$ pheO352 tyrA382 thi strR712 lacY1 xyl-5 & Baldwin \& Davidson (1981) \\
\hline JM109 & $\begin{array}{l}\text { recAl supE } 44 \text { end } A I \text { hsdRI7 gyrA96 relAl thi } \\
\triangle(\text { lac-pro } A B)\end{array}$ & Promega \\
\hline \multicolumn{3}{|l|}{ Plasmids } \\
\hline pHC79 & $A p^{R} T c^{R} c o s$ & Hohn \& Collins $(1980)$ \\
\hline pGEM-4Z & $\mathrm{Ap}^{\mathrm{R}}$ lac Z & Promega \\
\hline pGEM-5Zf (+) & $\mathrm{Ap}^{\mathrm{R}}$ lac Z & Promega \\
\hline pJF958 & $\mathrm{Phe}^{+}$clone from pHC79:PstI gene bank of JM300 & This study \\
\hline pJF9586 & $\begin{array}{l}\text { Phe }{ }^{+} 4.35 \mathrm{~kb} \text { PstI fragment subcloned from pJF958 into } \\
\text { pHC79 }\end{array}$ & This study \\
\hline pJF1954 & $\begin{array}{l}\text { Phe }^{+} 1477 \text { bp SphI/BalI fragment subcloned from } \\
\text { pJF9586 into pGEM-5Zf(+) }\end{array}$ & This study \\
\hline pJF1956 & $\begin{array}{l}788 \text { bp } S p h \mathrm{I} / E c o \text { RI fragment subcloned from } \\
\text { pJF1954 into pGEM-4Z }\end{array}$ & This study \\
\hline pJF1958 & $\begin{array}{l}581 \text { bp } S p h \mathrm{I} / E c o \mathrm{RV} \text { fragment subcloned from } \\
\text { pJF1954 into pGEM-5Zf(+) }\end{array}$ & This study \\
\hline pJF 1960 & $\begin{array}{l}711 \text { bp EcoRI/PstI fragment } \dagger \text { subcloned from } \\
\text { pJF1954 into pGEM-4Z }\end{array}$ & This study \\
\hline pJF1970 & $\begin{array}{l}896 \text { bp EcoRV/Ball fragment subcloned from } \\
\text { pJF1954 into pGEM-5Zf(+) }\end{array}$ & This study \\
\hline pJF1972 & $\begin{array}{l}775 \text { bp } S p h \mathrm{I} / \text { PstI fragment subcloned from } \\
\text { pJF9586 into pGEM-5Zf) +) }\end{array}$ & This study \\
\hline
\end{tabular}

* Escherichia coli Genetic Stock Center, Yale University.

$\dagger$ The PstI site was that of the multiple cloning region of pGEM-5Zf(+).

ensure that a full-length $p$ he $A$ gene would be among the clones. Partially Pst I methylated JM300 DNA fragments (size-fractionated to $10-20 \mathrm{~kb}$ on preparative agarose) were ligated into Pst I-digested pHC79 which had been treated with calf intestinal alkaline phosphatase. Recombinants from the ligation were packaged using a $\lambda$ DNA packaging kit, employing $2 \mathrm{~mm}$-putrescine to relax size selectivity. Approximately 2600 clones obtained by transfection of $E$. coli strain DH1 with the $\lambda$ packaging reaction mixture were screened for ability to transform the Phe $^{-}$P. stutzeri JM706 to prototrophy. Pools of $90-100$ clones were maintained on duplicate master plates of LB/tetracycline medium. For primary screening, small-scale plasmid isolations were prepared from the pooled clones present on each master plate. Transformation of JM706 to prototrophy with plasmid DNA isolated from the pooled clones was used to identify a pool yielding $\mathrm{Phe}^{+}$transformants. The original pool was divided into sub-pools and systematically screened to isolate individual clones. We isolated six clones which could be resolved by restriction analyses to reveal two clones differing only in some low- $M_{\mathrm{r}}$ Pst I fragments. These clones were characterized as described in Results.

Assay of P-protein activities. The methods for preparation of crude extracts from late-exponential cultures of $E$. coli and associated analytical procedures were previously described (Fischer et al., 1986). Chorismate mutase and prephenate dehydratase activities of the cloned phe $A$ gene product were assayed by the method of Cotton \& Gibson (1965) as modified by Ahmad \& Jensen (1988 $b$ ), except that substrate concentrations were increased to $2 \mathrm{~mm}$.

Biochemicals and chemicals. Unless indicated otherwise, all biochemicals and chemicals were obtained from Sigma. Barium prephenate was prepared from culture supernatants of a tyrosine auxotroph of Salmonella typhimurium (Dayan \& Sprinson, 1970) and was converted to the potassium salt with a twofold excess of $\mathrm{K}_{2} \mathrm{SO}_{4}$ before use. Chorismate was prepared from culture supernatants of Klebsiella pneumoniae (Gibson, 1964).

$D N A$ sequencing and analysis of sequence data. Specific restriction fragments were cloned into pGEM-4Z or pGEM-5Zf $(+)$ as shown in Fig. 2. Double-stranded plasmid DNA was sequenced in both directions by methodology using fluorescent chain-terminating dideoxy nucleotides (Prober et al., 1987) at the DNA Sequencing Core Laboratory (DSEQ) of the Interdisciplinary Center for Biotechnology Research (ICBR) at the University of Florida. The DSEQ operates a DNA sequencer (Genesis 2000 DNA Analysis System from DuPont). Primers to the bacteriophage SP6 or T7 RNA polymerase promoters, which flank the multiple cloning site of the pGEM vectors, were used to sequence in from the ends of each pGEM subclone. In order to sequence DNA beyond the range of the SP6 and T7 primers, 17-mer 


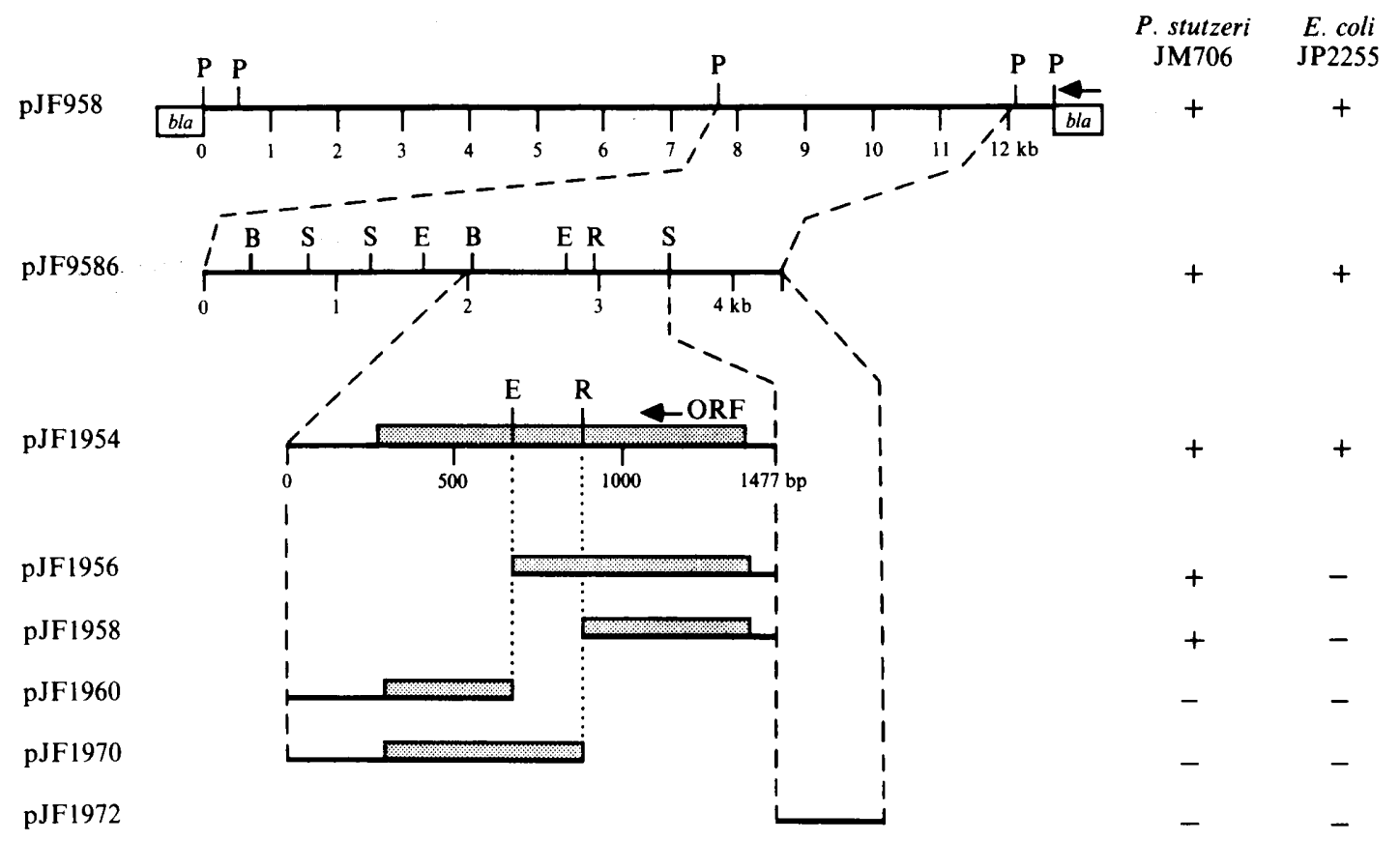

Fig. 2. Ability of specific restriction-generated fragments of $P$. stutzeri genomic DNA cloned in pHC79, pGEM-4Z or pGEM-5Z to transform or complement P-protein mutants of $P$. stutzeri and E. coli. The orientations of pJF958 and pJF9586 relative to the bla gene of pHC79 are depicted above. Uppercase letters show restriction sites in the $P$. stutzeri genomic DNA. P, Pst I ; B, BalI; S, SphI; E, EcoRI; $\mathrm{R}, E c o \mathrm{RV}$; ORF, open reading frame.

oligonucleotides were synthesized to regions upstream of the unsequenced regions at the DNA Synthesis Core Laboratory (DSYN) of ICBR. The DSYN employs an Applied Biosystems 380B synthesizer and a Pharmacia FPLC system for purification, quality control, and analysis of oligonucleotides.

The sequence data were analysed using the Biological Computing Facility (BCF) of ICBR. The BCF operates a MicroVax II, which was accessed through a dial-up modem. The programs and databases from the University of Wisconsin Genetics Computer Group (GCG) package (Devereux et al., 1984), available through the BCF, were used to analyse the DNA and deduced peptide sequences.

\section{Results}

Cloning of the P. stutzeri phe A gene in E. coli

A randomly generated $P s t$ I library of wild-type $P$. stutzeri DNA was constructed in E. coli, and approximately 2600 recombinants were screened by transformation of $P$. stutzeri JM706. Two plasmids were isolated which transformed JM706 to prototrophy. The presence of the structural gene for the P-protein in DNA sequences carried by these plasmids was confirmed by complementation of phe $A$ in $E$. coli JP2255. One plasmid, designated pJF958, was used to localize, subclone and sequence the $P$. stutzeri gene.

The strategy employed for subcloning and characterization of the P-protein gene carried on pJF958 is illustrated in Fig. 2. The two larger fragments (7.2 and $4.35 \mathrm{~kb}$ ) resulting from Pst I digestion of pJF958 were subcloned into pHC79. The $4.35 \mathrm{~kb}$ fragment, but not the $7.2 \mathrm{~kb}$ fragment, transformed $P$. stutzeri JM706 to prototrophy. Only one orientation of the $4.35 \mathrm{~kb}$ fragment in the bla gene of pHC79 was able to complement the P-protein mutation of JP2255, this orientation being the same as that of the original clone, pJF958. Thus, the cloned phe A gene appears to be dependent on pHC79 promoters for expression.

One plasmid carrying the $4.35 \mathrm{~kb}$ Pst I fragment, designated as pJF9586, was utilized for further subcloning. Southern blot hybridization (under moderate conditions of stringency) indicated that the ${ }^{32} \mathrm{P}$-labelled $4.35 \mathrm{~kb}$ Pst I fragment from pJF9586 hybridized with a $4.35 \mathrm{~kb}$ fragment of chromosomal DNA of $P$. stutzeri JM300 DNA digested with Pst I, whereas no hybridization was detected with Pst I-digested chromosomal DNA from wild-type $E$. coli $\mathrm{K} 12$ (data not shown).

The restriction sites shown in Fig. 2 were utilized to construct a set of subclones from pJF9586 in pGEM-4Z or pGEM-5Z for nucleotide sequencing. We found that a 1477 bp SphI-BalI fragment cloned into the SphI$E c o$ RV sites (within the multiple cloning region) of pGEM-5Z complemented the phe A mutation in $E$. coli JP2255. Disruption of the SphI-Ball fragment of this plasmid, pJF1954, at the EcoRV or EcoRI sites (yielding 
Table 2. Expression of the P. stutzeri pheA gene in E. coli JP2255

\begin{tabular}{|c|c|c|c|c|}
\hline \multirow{3}{*}{$\begin{array}{l}\text { Plasmid } \\
\text { in strain } \\
\text { JP2255* }\end{array}$} & \multirow{3}{*}{$\begin{array}{c}\text { Doubling } \\
\text { time } \\
\text { (min) }\end{array}$} & \multicolumn{3}{|c|}{ Specific activity† of: } \\
\hline & & \multirow{2}{*}{$\begin{array}{l}\text { Chorismate } \\
\text { mutase }\end{array}$} & \multicolumn{2}{|c|}{ Prephenate dehydratase } \\
\hline & & & $-\mathrm{Tyr}$ & + Tyr $\ddagger$ \\
\hline pHC79 & 66 & 0.0 & 0.0 & $0 \cdot 0$ \\
\hline pJF958 & 156 & $3 \cdot 7$ & $2 \cdot 5$ & $3 \cdot 8$ \\
\hline pJF9586 & 84 & $44 \cdot 3$ & $33 \cdot 6$ & $51 \cdot 2$ \\
\hline pGEM-5Z & 66 & $0 \cdot 0$ & 0.0 & 0.0 \\
\hline pJF1954 & 165 & $4 \cdot 1$ & $2 \cdot 8$ & 4.7 \\
\hline
\end{tabular}

* L-Tyrosine was supplemented to the growth medium of all cultures while L-phenylalanine was added only to the control cultures of JP2255(pHC79) and JP2255(pGEM-5Z).

$\dagger$ Specific activity is defined as nmol of phenylpyruvate produced $\min ^{-1}(\mathrm{mg} \text { protein })^{-1}$. The values for specific activity are representative of results of one of three independent repetitions.

$\ddagger L$-Tyrosine was added to the reaction mixture to give a final concentration of $0.5 \mathrm{~mm}$.

pJF1956, pJF1958, pJF1960 or pJF1970) resulted in loss of complementation of the phe $A$ defect. Neither chorismate mutase nor prephenate dehydratase activities were found in these transformed cells. Natural transformation of $P$. stutzeri JM706 with the restriction fragments shown in Fig. 2 revealed that the mutation responsible for the defective prephenate dehydratase is located within the 581 bp $S p h \mathrm{I}-E c o$ RV fragment.

\section{Expression of the P. stutzeri phe A gene in E. coli}

Results of enzymic assays of crude extracts prepared from E. coli JP2255 [a mutant which lacks both the Pprotein (chorismate mutase:prephenate dehydratase) and T-protein (chorismate mutase : prephenate dehydrogenase) activities] harbouring pJF958, pJF9586 or pJF1954 are summarized in Table 2. Chorismate mutase and prephenate dehydratase activities were demonstrated in cells transformed with each of these plasmids. The allosteric activation of prephenate dehydratase by Ltyrosine is an enzyme character state that typifies most Gram-negative bacteria (Byng et al., 1983), but not enteric bacteria. The enzyme activities given in Table 2 were determined from cultures grown in minimal medium so that the relative abilities of the three plasmids to confer independence of the nutritional requirement for phenylalanine could be estimated. Comparable specific activities were measured in extracts from cultures grown in rich medium (data not shown).

The growth rate of $E$. coli JP2255(pJF958) was still limited by phenylalanine, exhibiting a doubling time of more than $2.5 \mathrm{~h}$. This is consistent with the finding that specific activities for chorismate mutase and prephenate dehydratase in extracts of JP2255(pJF958) were only about $25 \%$ of the values determined for wild-type $E$. coli grown under comparable conditions (data not shown). Subcloning of the $4.35 \mathrm{~kb}$ Pst I fragment from pJF958 to yield JP2255(pJF9586) resulted in elevated expression levels which were about 12-fold greater than those obtained for JP2255(pJF958). The increased growth rate of JP2255(pJF9586) as compared to JP2255(pJF958) probably reflects the elevation in P-protein activities. When the 1477 bp SphI-BalI fragment was subcloned from pJF9586 into pGEM-5Z to yield pJF1954, the Pprotein activities were expressed at much lower levels. These lower P-protein activities are apparently due to vector differences (e.g. such as relative strengths of plasmid-encoded promoters) between $\mathrm{pHC79}$ and pGEM-5Z.

Western blot hybridization, employing antibody prepared to the P-protein from Acinetobacter calcoaceticus (Ahmad et al., 1988) (which cross-reacts strongly with the P-protein from $P$. stutzeri but poorly with the Pprotein from $E$. coli) was used to demonstrate the presence of protein bands in extracts of wild-type $P$. stutzeri and P-protein clones which have an apparent monomeric $M_{\mathrm{r}}$ of $41000-42000$ (data not shown).

\section{Sequence of the pheA gene}

Both strands were sequenced for the gene and surrounding regions except for the $100 \mathrm{bp}$ region upstream from the $S p h I$ site. The sequence shown in Fig. 3 contains an open reading frame (1095 base pairs) that encodes a protein of 365 amino acids with a calculated $M_{\mathrm{r}}$ of 40844 . Only one open reading frame among the six translation frames of the DNA sequence encoded a protein of sufficient $M_{\mathrm{r}}$ to be the $P$. stutzeri $\mathrm{P}$-protein. This reading frame was in agreement with the orientation of the cloned fragment required for expression in $E$. coli. The sequence GGAG, found 9 bp upstream from the start codon, is presumably the ribosome-binding site (Shine \& Dalgarno, 1974, 1975). The reading frame was terminated by the codon TAA. No prokaryotic factor-independent RNA polymerase terminators were detected (Brendel \& Trifonov, 1984).

\section{Discussion}

\section{Characteristics of the P. stutzeri pheA gene}

We have cloned, sequenced, and completed the initial characterization of the $P$. stutzeri phe $A$ gene. This is the first phenylalanine-pathway gene of a pseudomonad species to be cloned and sequenced. Within Superfamily 


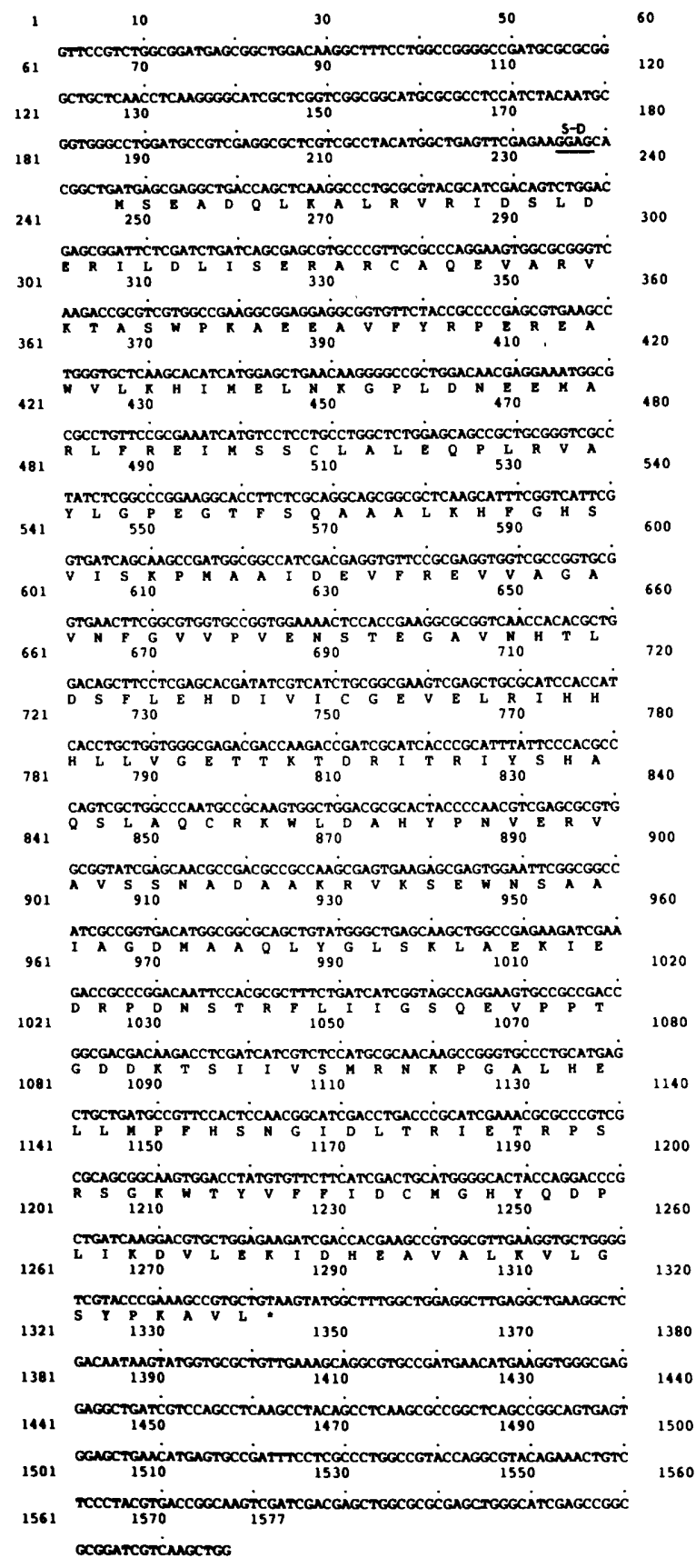

Fig. 3. Nucleotide sequence of the $P$. stutzeri phe $A$ gene and its flanking regions. The one-letter abbreviations for the deduced amino acid sequence are shown below the DNA sequence. The proposed Shine Dalgarno sequence (Shine \& Dalgarno, 1974, 1975) is underlined and labelled S-D.

B, P. stutzeri belongs to rRNA homology group I (Palleroni et al., 1973), which includes $P$. aeruginosa, $P$. fuorescens, $P$. putida and $P$. syringae. Group I pseudomonads exhibit considerable diversity for L-phenylalanine biosynthesis (Byng et al., 1983). Although the pathways for the monofunctional and bifunctional proteins catalysing the chorismate mutase and prephenate dehydratase reactions have been established through detailed enzymological characterization, and extensive evolutionary scenarios have been proposed (Ahmad \& Jensen, 1988a), little information about gene organization or molecular-genetic structure is available.

Enzymic activity in transformed cells harbouring the $4.35 \mathrm{~kb}$ Pst I fragment was observed in only one orientation (pJF9586) with respect to the vector pHC79, indicating that the phe $A$ gene was not efficiently expressed from native promoters in $E$. coli. Weak expression of $P$. stutzeri genes in $E$. coli due to inefficient recognition of promoters by $E$. coli RNA polymerase has been suggested (Fujita et al., 1989). Because the nucleotide sequence preceding the initiation codon at position 187 contains an apparent Shine-Dalgarno sequence (Shine \& Dalgarno, 1974, 1975) 9 bp upstream at bp 178, the mRNA of the $P$. stutzeri gene is expected to be translated in E. coli.

The $\mathrm{G}+\mathrm{C}$ content of the phe $A$ gene was $63.5 \mathrm{~mol} \%$, which falls within the published range of $60 \cdot 6-66 \cdot 3 \mathrm{~mol} \%$ $\mathrm{G}+\mathrm{C}$ for the $P$. stutzeri genome (Palleroni, 1984). Codon usage (data not shown) in the $P$. stutzeri phe $A$ gene resembles that in $P$. aeruginosa (West \& Iglewski, 1988), showing a marked preference for $G$ or $C$ in the third base in $87.4 \%$ of the codons. However, in contrast to all but one $P$. aeruginosa gene, $\mathrm{C}$ does not dominate as the nucleotide in the third codon position. Like phnB (Essar et al., 1990) [initially named as $\operatorname{trp} G$ (Crawford \& Eberly, 1986) in $P$. aeruginosa], $\mathrm{C}$ and $\mathrm{G}$ are used at nearly equal frequencies in the $P$. stutzeri phe $A$ gene. It is interesting to note that $p h n B$ encodes the $\beta$ subunit of an isoenzyme of anthranilate synthase which functions in pyocyanin synthesis and curiously has been found to resemble the TrpG subunit of the anthranilate synthase of $E$. coli more than the TrpG subunit expressed by $P$. aeruginosa. Whether the codon usage pattern shared by the $P$. stutzeri phe $A$ and $P$. aeruginosa phn $B$ genes portends a relationship of biological significance is unknown. Although the usage of rare codons in the phe $A$ gene is low, the tyrosine TAT codon was used equivalent with TAC, and TAA functioned as the termination codon instead of TGA, which is the preferred termination codon in $P$. aeruginosa genes (West \& Iglewski, 1988). The effects that these features of codon usage in the phe $A$ gene may have on expressivity of mRNA in vivo are questions for continuing investigation.

Comparison of the P. stutzeri and E. coli phe A genes and homology of their predicted gene products

Since the time that the nucleotide sequence of the $E$. coli phe $A$ gene was determined (Hudson \& Davidson, 1984), 


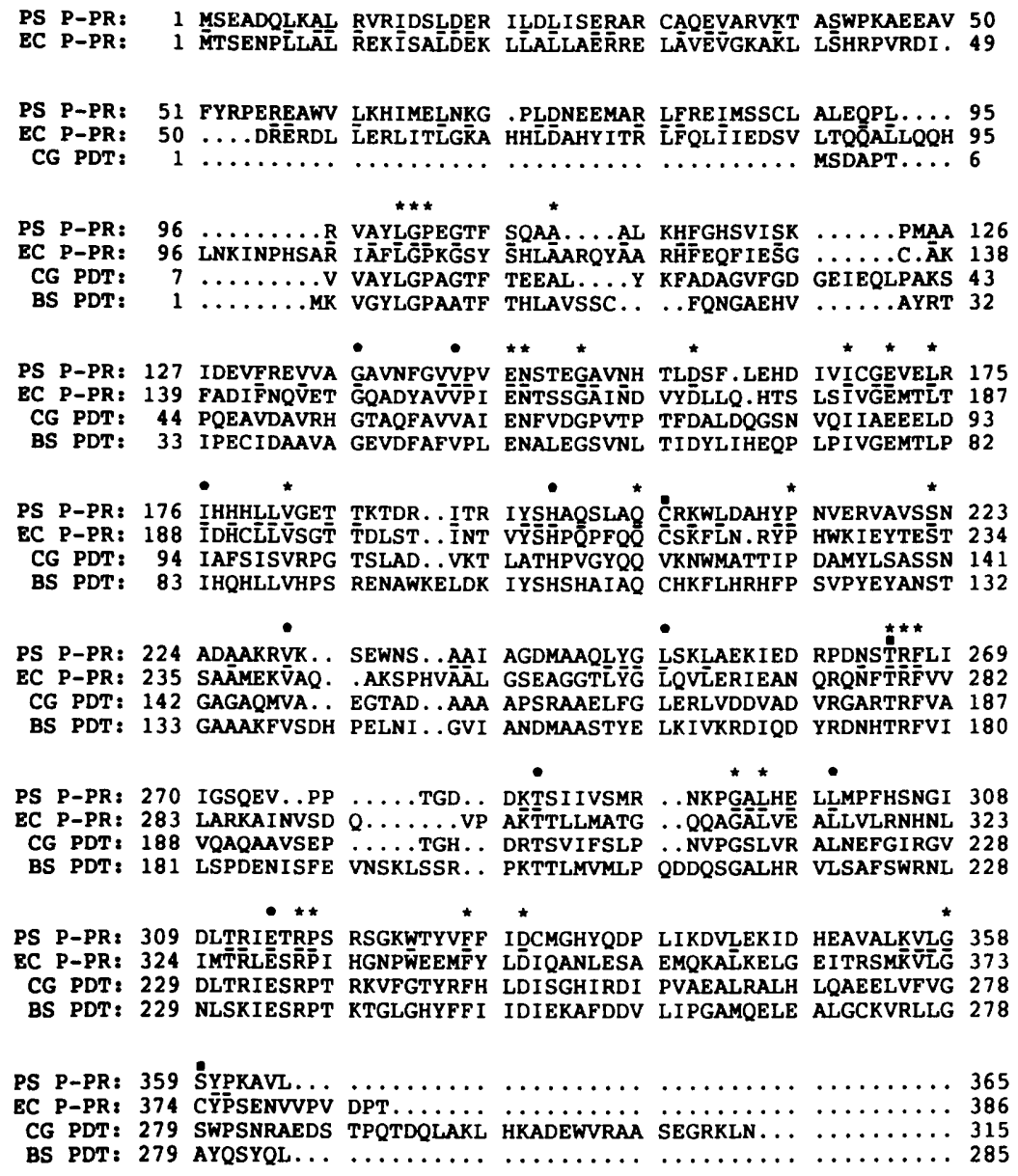

Fig. 4. Alignment of the deduced amino acid sequences of the $P$. stutzeri $\mathrm{P}$-protein, E. coli $\mathrm{P}$ protein, $C$. glutamicum PDT, and $B$. subtilis PDT. Gaps have been introduced to maximize alignments with respect to identical and functionally similar amino acids. Identity among amino acid residues of the P-proteins of $P$. stutzeri and $E$. coli is represented by short lines between the sequences. The positions of identical residues among all sequences are designated with asterisks. The locations of amino acids aligning with residues thought to be associated with the enzyme activities of the $E$. coli P-protein are indicated by . Numbers to the left and right of the sequences indicate amino acid residue positions. Abbreviations not previously defined: PS, P. stutzeri; EC, E. coli; CG, C. glutamicum; BS, B. subtilis; P-PR, P-protein. no other P-protein gene sequence has been reported. The deduced amino acid sequence encoded by the $P$. stutzer $i$ phe $A$ gene is comprised of 365 residues yielding an $M_{\mathrm{r}}$ of 40844. Although slightly smaller, the size of the $P$. stutzeri $\mathrm{P}$-protein falls within the same range as the $E$. coli P-protein, which is reported to consist of 386 amino acid residues having an $M_{\mathrm{r}}$ of 43111 (Hudson \& Davidson, 1984). Amino acid identities between the predicted phe $A$ gene products of $P$. stutzeri and $E$. coli are shown in Fig. 4. Alignment of the two amino acid sequences revealed that 114 of the 365 residues of the $P$. stutzeri gene product were identical to those of $E$. coli. Significant identity was noted throughout the entire sequence. From the group of three amino acid residues reported to be associated with the activities of the P-protein from E. coli (Baldwin \& Davidson, 1981; Hudson \& Davidson, 1984), the cysteine residue 216 and threonine residue 278 have been conserved in the $P$. stutzeri protein, while the cysteine residue 374 has undergone a change to serine. Interestingly, six residues immediately surrounding the cysteine residue at 374 have been conserved in both proteins.
Alignments of the amino acid sequences of the $P$. stutzeri $\mathrm{P}$-protein, E. coli $\mathrm{P}$-protein and $E$. coli T-protein revealed homology in the $\mathrm{N}$-terminal portions of these peptides (data not shown). Of the first 42 residues of the $P$. stutzeri P-protein, 18 and 14 were identical to the $E$. coli P-protein and T-protein, respectively. Similarly, alignments of the $E$. coli P-protein and T-protein peptides showed 18 amino acid matches for this region. The homology between the $E$. coli proteins and the $P$. stutzeri P-protein continues throughout the first $80-90$ residues of the $P$. stutzeri gene product but disappears beyond this region. This is consistent with the report by Hudson \& Davidson (1984) which demonstrated homology between only the $\mathrm{N}$-terminal portions (chorismate mutase domain) of the two bifunctional $E$. coli proteins.

In E. coli, the genes for the P-protein (pheA), T-protein $(t y r A)$, and DAHP synthase isoenzyme (tyrosine-sensitive) (aroF) are organized together in two operons on the chromosome, separated by a bidirectional transcription terminator (Hudson \& Davidson, 1984). In addition, unidentified reading frames (URF) were identified at 
regions outside of the two operons. The sequences $5^{\prime}$ and $3^{\prime}$ to the $P$. stutzeri phe $A$ gene were analysed for regions which might possess similarity to the phe $A$ and aroF/tyr $A$ operons of $E$. coli. After analysis with all six reading frames, no regions were recognized which demonstrated homology to the aroF gene product, the phe leader peptide, the $t y r A$ gene product, or the two URF regions. Experiments to recognize unidentified gene sequences in regions contiguous to the DNA sequenced for this investigation are in progress to reveal potential operons, and to establish gene order and organization.

\section{Evolutionary relationships between enzymes of phenylalanine biosynthesis}

In addition to the $E$. coli phe $A$, two genes encoding monofunctional PDTs have been sequenced from Grampositive bacteria. The deduced amino acid sequences of the monofunctional PDTs of Bacillus subtilis (Trach \& Hoch, 1989) and Corynebacterium glutamicum (Follettie \& Sinskey, 1986) showed strong identity to the Cterminal (residues 90-365) portion of the P. stutzeri $\mathrm{P}$ protein. The multiple alignment shown in Fig. 4 demonstrates that the peptide sequences of the $P$. stutzer $i$ P-protein, E. coli P-protein, C. glutamicum PDT and $B$. subtilis PDT are homologous. In this alignment 34 residues are conserved in all four peptides. Many more identities are seen if the sequences are compared in a pairwise fashion. The results obtained from binary alignments of the $P$. stutzeri peptide sequence with those of the E. coli P-protein, C. glutamicum PDT, or B. subtilis PDT (Table 3) reveal a limited range of identity values (31-35\%). Interestingly, the C. glutamicum and B. subtilis PDTs showed amino acid identities of $26 \%$ and $31 \%$, respectively, with the E. coli $\mathrm{P}$-protein sequence but only $29 \%$ when aligned with each other even though both enzymes are from Gram-positive bacteria, possessing similar allosteric characteristics of being stimulated by remote effectors (hydrophobic amino acids) and feedback inhibited by L-phenylalanine (Ahmad \& Jensen, 1986; Berry et al., 1987; Fazel \& Jensen, 1980; Pierson \& Jensen, 1974).

Of the three amino acid residues reported to be required for activity of the $E$. coli $\mathrm{P}$-protein (Baldwin \& Davidson, 1981; Hudson \& Davidson, 1984) and designated in Fig. 4, only the threonine at position 278 was conserved in the $P$. stutzeri P-protein, B. subtilis PDT, and $C$. glutamicum PDT. It is of interest to note that mutagenesis studies demonstrated that threonine residue 278 was essential for prephenate dehydratase activity (Baldwin \& Davidson, 1981). While it appears that the prephenate dehydratases may share a common ancestor, a monophyletic origin of chorismate mutase enzymes is not obvious since the CM-Fs of $B$. subtilis
Table 3. Comparison of binary alignments between various prephenate dehydratase enzymes

\begin{tabular}{lccc}
\hline \hline & \multicolumn{3}{c}{ Percentage identity/similarity } \\
\cline { 2 - 4 } \multicolumn{1}{c}{$\begin{array}{c}\text { Organism and } \\
\text { enzyme }\end{array}$} & $\begin{array}{c}\text { E. coli } \\
\text { P-protein }\end{array}$ & $\begin{array}{c}\text { C. glutamicum } \\
\text { PDT }\end{array}$ & $\begin{array}{c}\text { B. subtilis } \\
\text { PDT }\end{array}$ \\
\hline P. stutzeri P-protein & $31 / 57$ & $33 / 51$ & $35 / 58$ \\
C. glutamicum PDT & $26 / 48$ & & $29 / 55$ \\
B. subtilis PDT & $31 / 58$ & $29 / 55$ & \\
\hline \hline
\end{tabular}

(Gray et al., 1990) and Saccharomyces cerevisiae (Schmidheini et al., 1989) showed marginal to no similarity to the $P$. stutzeri P-protein, the E. coli $\mathrm{P}$-protein, the E. coli $\mathrm{T}$ protein, or to each other. The sequence of the gene encoding the bifunctional DAHP synthase : chorismate mutase from B. subtilis 168 (Hoch \& Nester, 1973; Nasser \& Nester, 1967) may provide clues about the evolution of chorismate mutase.

The phylogenetic relationships of Bacillus, Corynebacterium, Escherichia and Pseudomonas (rRNA homology group I) are not paralleled by the homology comparisons of the various chorismate mutase and prephenate dehydratase gene products considered in this paper. PDT (Bacillus and Corynebacterium), P-protein ( $P$. stutzeri, Escherichia) and T-protein (Escherichia) exhibit similar homologies in all combinations of pairwise comparisons. It would appear that the evolutionary time sufficient for maximal divergence has occurred between even the most closely related lineages compared (i.e. Escherichia and Pseudomonas). The similar level of homology retained for all dehydratase peptides presumably reflects a degree of conservation mandated by constraints linked to catalytic requirements for dehydratase activity. When sequence data are available for more closely related lineages, it will be interesting to see at what phylogenetic depth the molecular tree based upon dehydratase genes parallels the phylogenetic tree based upon $16 \mathrm{~S}$ rRNA.

This investigation was supported by National Institutes of Health Grant DK 38309. This paper is Florida Agricultural Experiment Station Journal no. R-01059.

\section{References}

Ahmad, S. \& Jensen, R. A. (1986). The evolutionary history of two bifunctional proteins that emerged in the purple bacteria. Trends in Biochemical Sciences 11, 108-112.

Ahmad, S. \& Jensen, R. A. (1988a). New prospects for deducing the evolutionary history of metabolic pathways in prokaryotes : aromatic biosynthesis as a case-in-point. Origins of Life 18, 41-57.

Ahmad, S. \& Jensen, R. A. (1988 $b$ ). Phylogenetic distribution of components of the overflow pathway to L-phenylalanine within the enteric lineage of bacteria. Current Microbiology 16, 295-302. 
Ahmad, S., Wilson, A. T. \& Jensen, R. A. (1988). Chorismate mutase:prephenate dehydratase from Acinetobacter calcoaceticus. Purification, properties and immunological cross-reactivity. European Journal of Biochemistry 176, 69-79.

Baldwin, G. S. \& Davidson, B. E. (1981). A kinetic and structural comparison of chorismate mutase/prephenate dehydratase from mutant strains of Escherichia coli $\mathrm{K}-12$ defective in the phe A gene. Archives of Biochemistry and Biophysics 211, 66-75.

Berry, A., Ahmad, S., Liss, A. \& Jensen, R. A. (1987). Enzymological features of aromatic amino acid biosynthesis reflect the phylogeny of mycoplasmas. Journal of General Microbiology 133, 2147-2154.

BRENDEL, V. \& TRIFONOV, E. N. (1984). A computer algorithm for testing potential prokaryotic terminators. Nucleic Acids Research 12, $4411-4427$.

BYNG, G. S. \& JENSEN, R. A. (1983). Impact of isozymes upon partitioning of carbon flow and regulation of aromatic biosynthesis in prokaryotes. In Isozymes, vol. 8, pp. 115-140. Edited by M. C. Ratazzi, J. G. Scandalios \& G. S. Whitt. New York: Alan R. Liss.

Byng, G. S., Whitaker, R. J. \& Jensen, R. A. (1983). Evolution of Lphenylalanine biosynthesis in rRNA homology group I of Pseudomonas. Archives of Microbiology 136, 163-168.

Carlson, C. A., Pierson, L. S., Rosen, J. J. \& Ingraham, J. L. (1983). Pseudomonas stutzeri and related species undergo natural transformation. Journal of Bacteriology 153, 93-99.

Carlson, C. A., Steenbergen, S. M. \& Ingraham, J. L. (1984). Natural transformation of Pseudomonas stutzeri by plasmids that contain cloned fragments of chromosomal deoxyribonucleic acid. Archives of Microbiology 140, 124-138.

Clewell, D. B. \& HelinsKi, D. R. (1969). Supercoiled circular DNAprotein complex in Escherichia coli: purification and induced conversion to an open circular form. Proceedings of the National Academy of Sciences of the United States of America 62, 11591166.

Cotton, R. G. H. \& Gibson, F. (1965). The biosynthesis of phenylalanine and tyrosine: enzymes converting chorismic acid into prephenic acid and their relationships to prephenate dehydratase and prephenate dehydrogenase. Biochimica et Biophysica Acta 100, 76-88.

Crawford, I. P \& Eberly, L. (1986). Structure and regulation of the anthranilate synthase genes in Pseudomonas aeruginosa. I. Sequence of $\operatorname{trp} G$ encoding the glutamine amidotransferase subunit. Molecular Biology and Evolution 3, 436-448.

DagerT, M. \& EhrLICH, S. D. (1979). Prolonged incubation in calcium chloride improves the competence of Escherichia coli cells. Gene 6, 23-28.

Davis, R. W., Botstein, D. \& Roth, J. R. (1980a). In Advanced Bacterial Genetics, pp. 201. Cold Spring Harbor. NY: Cold Spring Harbor Laboratory.

Davis, R. W., Thomas, M., Cameron, J., St John, T. P., Scherer, S. \& PADGETT, R. A. $(1980 \mathrm{~b})$. Rapid DNA isolations for enzymatic and hybridization analysis. Methods in Enzymology 65, 404-411.

DaYAN, J. \& SpRINSON, D. B. (1970). Preparation of prephenic acid. Methods in Enzymology 17A, 559-561.

Devereux, J., Haeberli, P. \& Smithies, O. (1984). A comprehensive set of sequence analysis programs for the VAX. Nucleic Acids Research 12, 387-395.

Essar, D. W., Eberly, L., Hadero, A. \& Crawford, I. P. (1990). Identification and characterization of genes for a second anthranilate synthase in Pseudomonas aeruginosa: interchangeability of the two anthranilate synthases and evolutionary implications. Journal of Bacteriology 172, 884-900.

FAZEL, A. M. \& JenSEN, R. A. (1980). Regulation of phephenate dehydratase in coryneform species of bacteria by L-phenylalanine and by remote effectors. Archives of Biochemistry and Biophysics 200, 165-176.

Fischer, R. S., Berry, A., Gaines, C. G. \& Jensen, R. A. (1986). Comparative action of glyphosate as a trigger of energy drain in eubacteria. Journal of Bacteriology 168, 1147-1154.

Follettie, M. T. \& Sinskey, A. J. (1986). Molecular cloning and nucleotide sequence of the Corynebacterium glutamicum phe $A$ gene. Journal of Bacteriology 167, 695-702.

Fujtta, M., Torigoe, K., Nakada, T., Tsusaki, K., Kubota, M., SaKaI, S. \& Tsujisaka, Y. (1989). Cloning and nucleotide sequence of the gene $(a m y P)$ for maltotetraose-forming amylase from Pseudomonas stutzeri MO-19. Journal of Bacteriology 171, 1333-1339.

GiBson, F. (1964). Chorismic acid : purification and some chemical and physical studies. Biochemical Journal 90, 256-257.

Gray, J. V., Golinelli-Pimpaneau, B. \& Knowles, J. R. (1990). Monofunctional chorismate mutase from Bacillus subtilis: purification of the protein, molecular cloning of the gene and overexpression of the gene product in Escherichia coli. Biochemistry 29, 376-383.

Hoch, J. A. \& Nester, E. W. (1973). Gene-enzyme relationships of aromatic acid biosynthesis in Bacillus subtilis. Journal of Bacteriology 116, 59-66.

HoHN, B. \& Collins, J. (1980). A small cosmid for efficient cloning of large DNA fragments. Gene 11, 291-298.

Hudson, G. S. \& Davidson, B. E. (1984). Nucleotide sequence and transcription of the phenylalanine and tyrosine operons of Escherichia coli K-12. Journal of Molecular Biology 180, 1023-1051.

Humphreys, G. O., Willshaw, G. A. \& ANDERson, E. S (1975). A simple method for the preparation of large quantities of pure plasmid DNA. Biochimica et Biophysica Acta 383, 457-463.

KuPERSZTOCH, Y. M. \& HelinsKI, D. R. (1973). A catenated DNA molecule as an intermediate in the replication of the resistance transfer factor R6K in Escherichia coli. Biochemical and Biophysical Research Communications 54, 1451-1459.

Maniatis, T., Fritsch, E. F. \& Sambrook, J. (1982). Molecular Cloning: a Laboratory Manual. Cold Spring Harbor, NY: Cold Spring Harbor Laboratory.

MilleR, J. H. (1972). Experiments in Molecular Genetics, pp. 431-435. Cold Spring Harbor, NY: Cold Spring Harbor Laboratory.

NASSER, D. \& NeSTER, E. W. (1967). Aromatic amino acid biosynthesis: gene-enzyme relationships in Bacillus subtilis. Journal of Bacteriology 94, 1706-1714.

Palleroni, N. J. (1984). Gram-negative aerobic rods and cocci. Family I. Pseudomonadaceae. In Bergeys Manual of Systematic Bacteriology, vol. 1, pp. 172-173. Edited by N. R. Krieg \& J. G. Holt. Baltimore: Williams \& Wilkins.

Palleroni, N. J., Kunisawa, R., Contopoulas, R. \& Doudoroff, M. (1973). Nucleic acid homologies in the genus Pseudomonas. International Journal of Systematic Bacteriology 23, 333-339.

Patel, N., Pierson, D. L. \& Jensen, R. A. (1977). Dual enzymatic routes to L-tyrosine and L-phenylalanine via pretyrosine in Pseudomonas aeruginosa. Journal of Biological Chemistry 252, 5839-5846.

Pierson, D. L. \& Jensen, R. A. (1974). Metabolic interlock: control of an interconvertible prephenate dehydratase by hydrophobic amino acids in Bacillus subtilis. Journal of Molecular Biology 90, 563-579.

Prober, J. M., Trainor, G. L., Dam, R. J., Hobbs, F. W., Robertson, C. W., Zagursky, R. J., Cocuzza, A. J., Jensen, M. A. \& BAUMEISTER, K. (1987). A system for rapid DNA sequencing with fluorescent chain-terminating dideoxy nucleotides. Science $\mathbf{2 3 8}$, 336-341

Schmidheini, T., Sperisen, P., Paravicini, G., Hutter, R. \& Braus, G. (1989). A single point mutation results in a constitutively activated and feedback-resistant chorismate mutase of Saccharomyces cerevisiae. Journal of Bacteriology 171, 1245-1253.

Shilo, I. \& Sugimota, S. (1979). Two components of chorismate mutase in Brevibacterium flavum. Journal of Biochemistry 86, 17-25.

SHINE, J. \& Dalgarno, L. (1974). The 3'-terminal sequence of Escherichia coli $16 \mathrm{~S}$ ribosomal RNA : complementarity to nonsense triplets and ribosome binding sites. Proceedings of the National Academy of Sciences of the United States of America 71, 1342-1346.

Shine, J. \& Dalgarno, L. (1975). Determination of cistron specificity in bacterial ribosomes. Nature, London 254, 34-38.

Trach, K. \& HосH, J. A. (1989). The Bacillus subtilis spo0B stage 0 sporulation operon encodes an essential GTP-binding protein. Journal of Bacteriology 171, 1362-1371.

WEST, S. E. H. \& IGLEWSKI, B. H. (1988). Codon usage in Pseudomonas aeruginosa. Nucleic Acids Research 16, 9323-9335.

YUAN, R. \& LIN, Y. (1982). The purification of restriction enzymes and DNA substrates. Their use in DNA sequencing. In Genetic Engineering Techniques: Recent Developments, pp. 337-349. Edited by P. C. Huang, T. T. Kuo \& R. Wu. New York: Academic Press.

Zamir, L. O., Tiberio, R., Fiske, M., Berry, A. \& Jensen, R. A. (1985). Enzymatic and nonenzymatic dehydration reactions of Larogenate. Biochemistry 24, 1607-1612. 